\title{
Synthesis of activated spirocyclopentanes via a cascade Michael/alkylation reaction of ethyl-4-chloro-3-oxobutanoate and 2- arylidene-1,3-indandiones
}

\author{
Nian-hua Luo, ${ }^{\text {a* }}$ Da-gui Zheng, ${ }^{\text {a }}$ Xue-jing Zhang, ${ }^{\text {b }}$ and Ming Yan ${ }^{\text {b }}$ \\ ${ }^{a}$ Key Laboratory of Applied Organic Chemistry, Higher Institutions of Jiangxi Province, School \\ of Chemistry and Chemical Engineering, Shangrao Normal University, Shangrao 334001, China \\ ${ }^{b}$ Institute of Drug Synthesis and Pharmaceutical Process, School of Pharmaceutical Sciences, \\ Sun Yat-sen University, Guangzhou 510006, China \\ E-mail: luoxiaoge102@,163.com
}

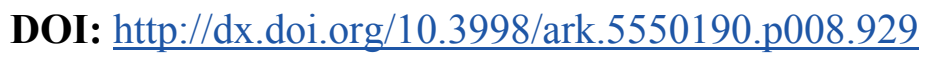

\begin{abstract}
A cascade Michael/alkylation reaction of ethyl-4-chloro-3-oxobutanoate and 2-Arylidene-1,3indandiones had been studied, providing a number of activated spirocyclopentanes in excellent yields (up to 96\%) and diastereoselectivities (up to dr > 20:1). Different bases were evaluated and triethylamine was found to be the most efficient for this transformation under mild reaction.
\end{abstract}

Keywords: Cascade reaction, spirocyclopentanes, bases

\section{Introduction}

2-Arylidene-1,3-indandiones are mostly attractive Michael acceptors ${ }^{1-5}$ for the resulted substituted 1,3-indandiones had been widely found in many natural products with useful biological activities (Scheme 1). ${ }^{6-11}$ Among various 1,3-indandiones and their derivatives, multicyclic spiro-1,3-indandiones are especially valuable. For example, fredericamycin $\mathbf{A}$ was reported as an antitumor compound with antibiotic properties. ${ }^{8}$ Spiroheterocyclic dihydropyrrolo[2,1-a]isoquinolines B had potential pharmacological effects such as sedative, hypotensive and neuromuscular blocking activities. ${ }^{9}$ Biphenyl-based spirocyclic ketones $\mathbf{C}$ was widely used as new anticancer agents. ${ }^{11}$ Among the chemical synthesis methods of these useful bioactive compounds, the cascade reactions based on 1,3-indandione and its derivatives are extremely attractive in terms of efficiency and atomic economy. ${ }^{12-15}$ For example, Barbas III and co-workers developed a multicomponent reaction through combinations of Aldol, Wittig, Knoevenagel, Michael, Diels-Alder and Huisgen cycloaddition reactions, providing polycyclic spirotriones in good yields and diastereoselectivities. ${ }^{12} \mathrm{Li}$ and co-workers reported a cascade 
reaction of 1,3-indanedione for the synthesis of tricyclic spiro-1,3-indandiones. ${ }^{13}$ Ramachary and co-workers developed a cascade reaction of 2-Arylidene-indan-1,3-diones to synthesize drug-like cyclohexanes. ${ }^{14}$ Other examples such as the Knoevenagel/Diels-Alder/ epimerization reaction of 1,3-indandione were also reported. ${ }^{15}$

Despite the extensive efforts have been made, the synthesis of all carbon spiro-1,3indandiones still presents a big challenge in organic synthesis. ${ }^{16-18}$ Recently, we have been interested in the organocatalytic synthesis of cyclic products via cascade/domino reactions. ${ }^{19-27}$ Herein, we report the cascade Michael/Alkylation reaction of ethyl-4-chloro-3-oxobutanoate and 2-Arylidene-1,3-indandiones, which provided activated spirocyclopentanes in excellent yields and diastereoselectivities.

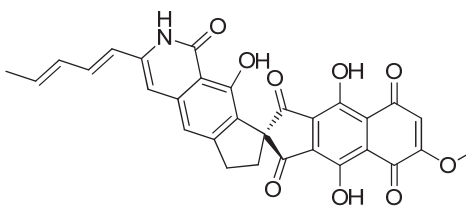

Fredericamycin A

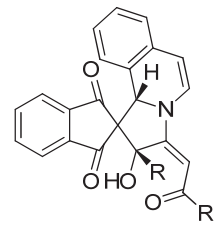

B

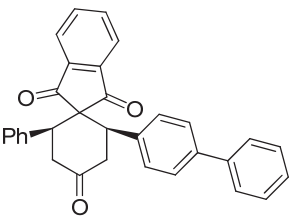

C

Scheme 1. Multicyclic spiro-1,3-indandiones.

\section{Results and Discussion}

Firstly, the cascade Michael/Alkylation reactions of 2-Arylidene-1,3-indandiones 1a and ethyl-4chloro-3-oxobutanoate 2 were examined in $\mathrm{CH}_{2} \mathrm{Cl}_{2}$ at room temperature with different bases as the catalysts (Table 1). Initial screening of the reaction conditions demonstrated that the organic and inorganic base had a significant role to play in both reactivity and selectivity. Using the inorganic bases as the catalysts, the cascade Michael/Alkylation product 3a was obtained in low yields and diastereoselectivities (Table 1, entries 1-8). The spirocyclopentane 3a was achieved in good yields and diastereoselectivities by using the organic bases as the catalysts (Table 1, entries 9-12). Further investigation demonstrated that the $\mathrm{Et}_{3} \mathrm{~N}$ was preferred in terms of the yield and diastereoselectivity (Table 1, entry 12). When the catalyst loading of $\mathrm{Et}_{3} \mathrm{~N}$ was used to be 200 mol \%, the highest yield (96\%) and diastereoselectivity (95:5) was obtained (Table 1, entry 13). 
Table 1. Base-catalyzed cascade Michael/Alkylation reaction of $\mathbf{1 a}$ with $\mathbf{2}^{\mathrm{a}}$

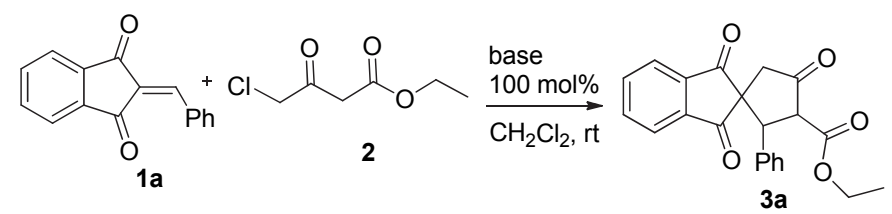

\begin{tabular}{ccccc}
\hline Entry & Base & Time $(\mathrm{h})$ & $\mathrm{Dr}^{\mathrm{b}}$ & ${\text { Yield }(\%)^{\mathrm{c}}}^{\mathrm{c}}$ \\
\hline 1 & $\mathrm{KOH}$ & 6 & $60: 40$ & 32 \\
2 & $\mathrm{NaOH}$ & 6 & $61: 39$ & 36 \\
3 & $\mathrm{~K}_{2} \mathrm{CO}_{3}$ & 8 & $65: 35$ & 41 \\
4 & $\mathrm{KHCO}_{3}$ & 8 & $64: 36$ & 35 \\
5 & $\mathrm{Na}_{2} \mathrm{CO}_{3}$ & 8 & $68: 32$ & 38 \\
6 & $\mathrm{NaHCO}_{3}$ & 8 & $65: 35$ & 31 \\
7 & $\mathrm{LiOAc}$ & 24 & $70: 30$ & 42 \\
8 & $\mathrm{NaOAc}$ & 24 & $70: 30$ & 33 \\
9 & $\mathrm{DABCO}$ & 24 & $86: 14$ & 85 \\
10 & $\mathrm{DBU}$ & 4 & $85: 15$ & 84 \\
11 & $\mathrm{DMAP}$ & 6 & $88: 12$ & 86 \\
12 & $\mathrm{Et} \mathrm{H}_{3} \mathrm{4}$ & 4 & $95: 5$ & 91 \\
$13^{\mathrm{d}}$ & $\mathrm{Et}_{3} \mathrm{~N}$ & 2 & $95: 5$ & 96 \\
\hline
\end{tabular}

${ }^{a}$ Reactions were carried out with $1 \mathrm{a}(0.1 \mathrm{mmol}), 2(0.12 \mathrm{mmol})$ and catalyst $(0.1 \mathrm{mmol})$ in $\mathrm{CH}_{2} \mathrm{Cl}_{2}(1 \mathrm{~mL})$ at room temperature.

${ }^{\mathrm{b}}$ Determined by ${ }^{1} \mathrm{H}$ NMR analysis of the crude product.

${ }^{\mathrm{c}}$ Isolated yields. ${ }^{\mathrm{d}} 200 \% \mathrm{~mol} \mathrm{Et}_{3} \mathrm{~N}$ was added.

To get a better reaction conditions, we next screened the effects of solvents (Table 2, entries 1-8). Among the solvents tested, $\mathrm{CHCl}_{3}$ was found to be the best solvent to give the best yield and selectivity (Table 2, entries 7 and 8). A slightly lower yields but also excellent diastereoselectivity were observed with the solvents of $\mathrm{CH}_{2} \mathrm{Cl}_{2}$ (Table 2, entry 6). Almost the same selectivities were obtained when reactions were performed at the solvents of acetone, THF and toluene (Table 2, entries 3-5). Reactions in $\mathrm{MeOH}$ or DMF afforded the desired product 3a in only low yield and selectivity (Table 2, entries 1 and 2). 
Table 2. Screening of the solvent and temperature ${ }^{a}$

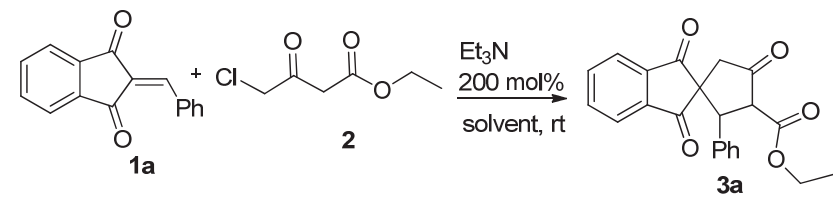

\begin{tabular}{ccccc}
\hline Entry & Solvent & Time $(\mathrm{h})$ & $\operatorname{Dr}^{\mathrm{b}}$ & ${\text { Yield }(\%)^{\mathrm{c}}}^{\mathrm{c}}$ \\
\hline 1 & $\mathrm{MeOH}$ & 24 & $70: 30$ & 38 \\
2 & DMF & 24 & $68: 32$ & 29 \\
3 & Acetone & 12 & $82: 18$ & 76 \\
4 & THF & 12 & $80: 20$ & 69 \\
5 & Toluene & 12 & $85: 15$ & 90 \\
6 & $\mathrm{CH}_{2} \mathrm{Cl}_{2}$ & 2 & $95: 5$ & 96 \\
7 & $\mathrm{CHCl}_{3}$ & 2 & $98: 2$ & 98 \\
$8^{\mathrm{d}}$ & $\mathrm{CHCl}_{3}$ & 12 & $98: 2$ & 95 \\
\hline
\end{tabular}

${ }^{\mathrm{a}}$ Reactions were carried out with $1 \mathrm{a}(0.1 \mathrm{mmol}), 2(0.12 \mathrm{mmol})$ and $\mathrm{Et}_{3} \mathrm{~N}(0.2 \mathrm{mmol}, 56 \mathrm{uL})$ in solvent $(1 \mathrm{~mL})$ at RT.

${ }^{\mathrm{b}}$ Determined by ${ }^{1} \mathrm{H}$ NMR analysis of the crude product.

${ }^{\mathrm{c}}$ Isolated yields. ${ }^{\mathrm{d}}$ The reaction was carried out at $0{ }^{\circ} \mathrm{C}$.

Under the optimized reaction condition, the $\mathrm{Et}_{3} \mathrm{~N}$ as base and $\mathrm{CHCl}_{3}$ as the solvent were proved to be efficient for the synthesis of spirocyclopentanes (Table 3). For example, spirocyclopentanes $\mathbf{3}$ were obtained in excellent yields and diastereoselectivities by using different substrates such as aryl and heteroaryl-1,3-indandiones. The position of the substituents at the phenyl ring seems to have slightly effect on the yields and diastereoselectivities. As can be seen in table 3, the para-substitution generally resulted in better yields and diastereoselectivities, no matter electron-withdrawing or electron-donating groups were introduced (Table 3, entries 4, 6-8). In comparison, ortho-chloro and meta-chloro substituted 2-Arylidene-1,3-indandiones $\mathbf{1 b}$ and 1c afforded lower yields and diastereoselectivities (Table 3, entries 2-3). Similarly, orthomethoxy substituted 2-arylidene-1,3-indandione 1e gave lower yields and diastereoselectivities than its para-substituted analog $\mathbf{1 f}$ (Table 3, entries 5 and 6). The 2-thiophenyl-1,3-indandione 1i provided the product in lower yield (91\%, Table 3, entry 9). Disappointedly, no Michael/Alkylation product was obtained when the 2-furyl-1,3-indandione was used in the reaction. 
Table 3 Synthesis of spirocyclopentanes 3 from a variety of 1,3-indandiones ${ }^{\mathrm{a}}$

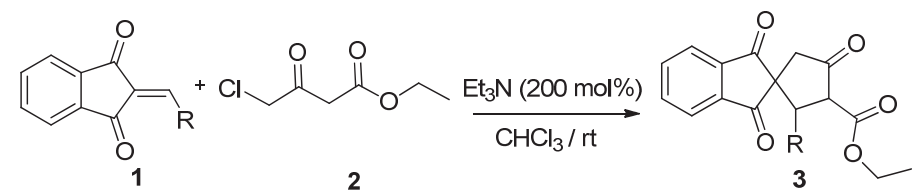

\begin{tabular}{|c|c|c|c|}
\hline Entry & $\mathrm{R}$ & Yield $(\%)^{b}$ & $\mathrm{Dr}^{\mathrm{c}}$ \\
\hline 1 & $\mathrm{Ph}(\mathbf{1 a})$ & 98 & $98: 2$ \\
\hline 2 & $2-\mathrm{Cl}-\mathrm{C}_{6} \mathrm{H}_{4}(\mathbf{1 b})$ & 94 & $96: 4$ \\
\hline 3 & $3-\mathrm{Cl}-\mathrm{C}_{6} \mathrm{H}_{4}(\mathbf{1 c})$ & 90 & $95: 5$ \\
\hline 4 & $4-\mathrm{Cl}-\mathrm{C}_{6} \mathrm{H}_{4}(\mathbf{1 d})$ & 98 & $98: 2$ \\
\hline 5 & $2-\mathrm{MeO}-\mathrm{C}_{6} \mathrm{H}_{4}(\mathbf{1 e})$ & 93 & $96: 4$ \\
\hline 6 & $4-\mathrm{MeO}-\mathrm{C}_{6} \mathrm{H}_{4}$ (1f) & 97 & $98: 2$ \\
\hline 7 & $4-\mathrm{F}-\mathrm{C}_{6} \mathrm{H}_{4}(\mathbf{1 g})$ & 98 & $98: 2$ \\
\hline 8 & $4-\mathrm{Br}^{-} \mathrm{C}_{6} \mathrm{H}_{4}(\mathbf{1 h})$ & 96 & $97: 3$ \\
\hline $9^{d}$ & 2-thionyl (1i) & 91 & $96: 4$ \\
\hline 10 & 2-furyl & - & - \\
\hline
\end{tabular}

${ }^{\mathrm{a}}$ Reactions were carried out with $1(0.1 \mathrm{mmol}), 2(0.12 \mathrm{mmol})$ and $\mathrm{Et}_{3} \mathrm{~N}(0.2 \mathrm{mmol})$ in $\mathrm{CHCl}_{3}(1$ $\mathrm{mL}$ ) at $\mathrm{RT}$ for $2 \mathrm{~h}$.

${ }^{\mathrm{b}}$ Isolated yields. ${ }^{\mathrm{c}}$ Determined by ${ }^{1} \mathrm{H}$ NMR.

${ }^{\mathrm{d}}$ The reaction was carried out at room temperature for $8 \mathrm{~h}$.

After the success of Michael/Alkylation reaction of ethyl-4-chloro-3-oxobutanoate, the reaction of ethyl-4-bromo-3-oxobutanoate with 2-Arylidene-1,3-indandione 1a was also studied. Disappointedly, only moderate yield and diastereoselectivity were obtained in comparison with ethyl-4-chloro-3-oxobutanoate (Scheme 2). Further studies are in progress in our group.
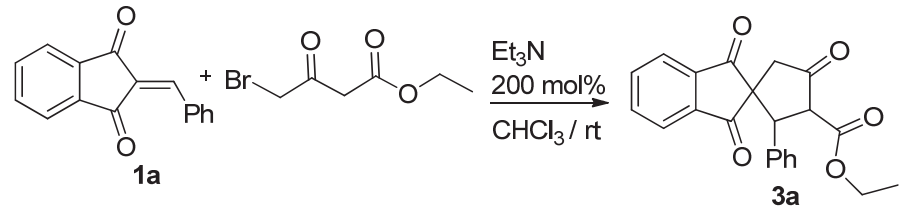

$72 \%$ yield, $65: 35 \mathrm{dr}$

Scheme 2. Reaction of ethyl-4-bromo-3-oxobutanoate and 2-Arylidene-1,3-indandione 1a.

The product 3a could be readily decarboxylationed by concentrated hydrochloric acid. The treatment of 3a with concentrated hydrochloric acid in water provided activated spirocyclopentane 4 in excellent yield and diastereoselectivity (Scheme 3). 


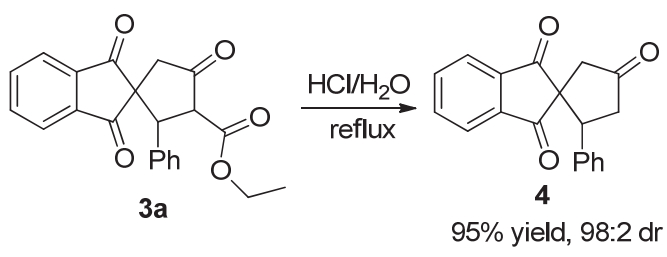

Scheme 3. Transformation of 3a to spirocyclopentane 4.

An asymmetric version of this reaction was also studied by using diphenyl-L-prolinol as the catalyst, but only moderate yield and low enantioselectivity were achieved (Scheme 4). ${ }^{18}$ Further studies are also in progress in our group.

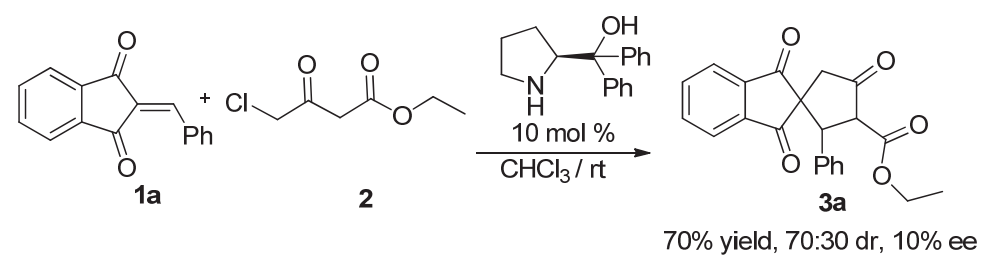

Scheme 4. Asymmetric reaction of Ethyl-4-chloro-3-oxobutanoate and 2-Arylidene-1,3indandione.

A proposed mechanism for the cascade Michael/Alkylation reaction is illustrated in Scheme $5{ }^{6}$ The possible catalytic Michael/Alkylation reaction may go through three main steps: (a) the deprotonation of ethyl-4-chloro-3-oxobutanoate by triethylamine gives the alpha-carbon anion; (b) the Michael addition of the ethyl-4-chloro-3-oxobutanoate to 2-Arylidene-1,3-indandiones; (c) intramolecular cyclization afforded spirocyclopentanes $\mathbf{3 a}$ in excellent yield.

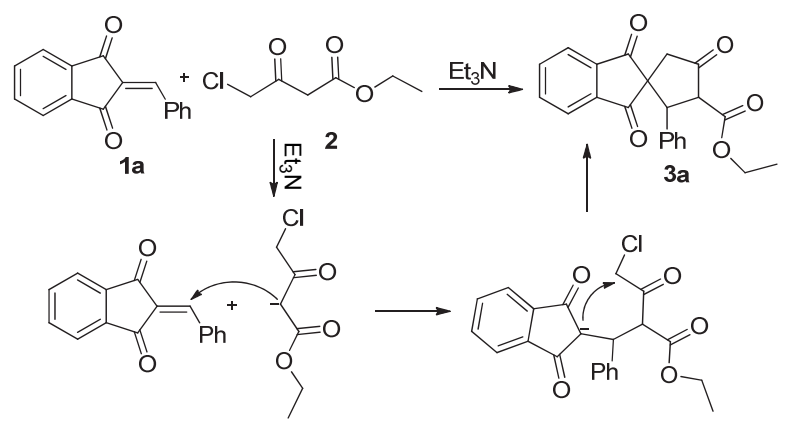

Scheme 5. Possible mechanism for the cascade Michael/alkylation reaction. 


\section{Conclusions}

We have developed a cascade Michael/Alkylation reaction of ethyl-4-chloro-3-oxobutanoate and 2-Arylidene-1,3-indandiones, providing a number of activated spirocyclopentanes in excellent yields (up to $96 \%$ ) and diastereoselectivities (up to $\mathrm{dr}>20: 1$ ).

\section{Experimental Section}

General. ${ }^{1} \mathrm{H}$ NMR and ${ }^{13} \mathrm{C}$ NMR spectra were recorded on Bruker AVANCE 400 spectrometer. Chemical shifts of protons are reported in parts per million downfield from tetramethylsilane $(\delta$ $=0)$. Chemical shifts of carbon are referenced to the central peak of the solvent $\left(\mathrm{CDCl}_{3}, \delta=\right.$ 77.0). Peaks are labeled as singlet (s), doublet (d), triplet (t), quartet (q) and multiplet (m). Optical rotations were measured on a Perkin-Elmer 341 digital polarimeter. Melting points were measured on a WRS-2A melting point apparatus and are uncorrected. The high resolution mass spectroscopic data were obtained with Shimadzu LCMS-IT-TOF spectrometer. Infrared (IR) spectra were recorded on a Bruker Tensor 37 spectrophotometer. Data are represented as follows: frequency of absorption $\left(\mathrm{cm}^{-1}\right)$, intensity of absorption $(\mathrm{s}=$ strong, $\mathrm{m}=$ medium, $\mathrm{w}=$ weak). Enantiomeric excesses were determined by HPLC using a Daicel Chiralpak OD-H column and eluting with a hexane/i-PrOH solution. Flash chromatography was performed over silica gel (230-400 mesh), purchased from Qingdao Haiyang Chemical Co., Ltd. Commercially available reagents and analytical grade solvents were used without further purification. 2Arylidene-1,3-indandiones were prepared according to reported procedures. ${ }^{28}$

\section{Typical procedure for asymmetric synthesis of spirocyclopentanes}

A solution of $1 \mathrm{a}(23.4 \mathrm{mg}, 0.1 \mathrm{mmol})$ and $2(16.4 \mathrm{mg}, 0.12 \mathrm{mmol})$ in $\mathrm{CHCl}_{3}(1 \mathrm{~mL})$ was stirred at room temperature for $10 \mathrm{~min}$. Then, $\mathrm{Et}_{3} \mathrm{~N}(56 \mathrm{uL})$ was added. The reaction solution was stirred at room temperature for $2 \mathrm{~h}$. Then, the solvent was evaporated under vacuum, and the residue was purified by flash column chromatography over silica gel (petroleum ether/EtOAc 3/1) to give product 3a as a white solid.

Ethyl 1',3',4-trioxo-2-phenyl-1',3'-dihydrospiro[cyclopentane-1,2'-indene]-3-carboxylate (3a). White solid, mp 134.5-135.6 ${ }^{\circ} \mathrm{C} ;{ }^{1} \mathrm{H}$ NMR $\left(400 \mathrm{MHz}, \mathrm{CDCl}_{3}\right) \delta$ : 7.89-7.88 (m, 1H), 7.73$7.70(\mathrm{~m}, 3 \mathrm{H}), 7.06-7.03(\mathrm{~m}, 5 \mathrm{H}), 4.46(\mathrm{~d}, J 13.6 \mathrm{~Hz}, 1 \mathrm{H}), 4.40(\mathrm{~d}, J 13.6 \mathrm{~Hz}, 1 \mathrm{H}), 4.21-4.11(\mathrm{~m}$, 2H), $2.99(\mathrm{~d}, J 18.4 \mathrm{~Hz}, 1 \mathrm{H}), 2.69(\mathrm{~d}, J 18.4 \mathrm{~Hz}, 1 \mathrm{H}), 1.23-1.20(\mathrm{~m}, 3 \mathrm{H}) ;{ }^{13} \mathrm{C}$ NMR $(100 \mathrm{MHz}$, $\left.\mathrm{CDCl}_{3}\right) \delta: 205.0,201.9,199.9,167.4,142.3,141.5,136.1,136.0,133.7,128.6,128.1,127.5$, 123.2, 123.1, 61.8, 60.0, 57.7, 53.0, 43.8, 14.1; IR (thin film) $v / \mathrm{cm}^{-1}: 1706(\mathrm{w}), 1599$ (s), 1562 (s), $1432(\mathrm{~m}), 1384$ (s), 1075 (m); HRMS (ESI) calcd for $\mathrm{C}_{22} \mathrm{H}_{18} \mathrm{NaO}_{5}(\mathrm{M}+\mathrm{Na})^{+}$: 385.1046, found: 385.1041 .

Ethyl-2-(2-chlorophenyl)-1',3',4-trioxo-1',3'-dihydrospiro[cyclopentane-1,2'-indene]-3- 
carboxylate (3b). White solid, mp 132.9-134.7 ${ }^{\circ} \mathrm{C}$; ${ }^{1} \mathrm{H}$ NMR (400 MHz, CDCl3) $\delta: 7.93$ (d, J 7.6 $\mathrm{Hz}, 1 \mathrm{H}), 7.76-7.62(\mathrm{~m}, 3 \mathrm{H}), 7.24-7.20(\mathrm{~m}, 1 \mathrm{H}), 7.08-7.05(\mathrm{~m}, 2 \mathrm{H}), 6.96(\mathrm{t}, J 7.6 \mathrm{~Hz}, 1 \mathrm{H}), 5.07$ $(\mathrm{d}, J 13.6 \mathrm{~Hz}, 1 \mathrm{H}), 4.30(\mathrm{~d}, J 13.2 \mathrm{~Hz}, 1 \mathrm{H}), 4.18-4.11(\mathrm{~m}, 2 \mathrm{H}), 3.12(\mathrm{~d}, J 18.8 \mathrm{~Hz}, 1 \mathrm{H}), 2.76(\mathrm{~d}, J$

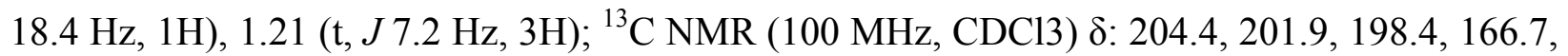
$142.3,141.3,136.1,136.0,134.8,131.8,130.0,129.1,128.0,126.9,123.4,122.9,61.9,59.8$, 59.2, 47.9, 42.9, 14.0; IR (thin film) $v / \mathrm{cm}^{-1}: 2925(\mathrm{w}), 1598(\mathrm{~s}), 1561(\mathrm{~s}), 1433(\mathrm{~m}), 1384(\mathrm{~s})$, 1077 (m), 719 (m); HRMS (ESI) calcd for $\mathrm{C}_{22} \mathrm{H}_{17} \mathrm{ClNaO}_{5}(\mathrm{M}+\mathrm{Na})^{+}: 419.0657$, found: 419.0661 .

Ethyl-2-(3-chlorophenyl)-1',3',4-trioxo-1',3'-dihydrospiro[cyclopentane-1,2'-indene]-3carboxylate (3c). White solid, mp 139.2-140.8 ${ }^{\circ} \mathrm{C} ;{ }^{1} \mathrm{H} \mathrm{NMR}\left(400 \mathrm{MHz}, \mathrm{CDCl}_{3}\right) \delta$ : 7.92 (d, J 7.6 $\mathrm{Hz}, 1 \mathrm{H}), 7.78-7.74(\mathrm{~m}, 3 \mathrm{H}), 7.05-6.96(\mathrm{~m}, 4 \mathrm{H}), 4.41$ (d, $J 13.6 \mathrm{~Hz}, 1 \mathrm{H}), 4.36(\mathrm{~d}, J 13.6 \mathrm{~Hz}, 1 \mathrm{H})$, 4.20-4.15 (m, 2H), 2.98 (d, J 18.4 Hz, 1H), 2.69 (d, J 18.4 Hz, 1H), $1.24(\mathrm{t}, J 7.2 \mathrm{~Hz}, 3 \mathrm{H}) ;{ }^{13} \mathrm{C}$ $\operatorname{NMR}\left(100 \mathrm{MHz}, \mathrm{CDCl}_{3}\right) \delta:$ 204.2, 201.6, 199.5, 167.1, 142.2, 141.4, 136.4, 136.3, 135.9, 134.6, $129.9,128.4,127.8,125.7,123.3,123.2,61.9,59.7,57.6,52.2,43.9,41.1$; IR (thin film) $v / \mathrm{cm}^{-1}$ : 2926 (w), 1706(w), 1597(s), 1561 (s), 1434 (m), 1384 (s), 1076 (m); HRMS (ESI) calcd for $\mathrm{C}_{22} \mathrm{H}_{17} \mathrm{ClNaO}_{5}(\mathrm{M}+\mathrm{Na})^{+}:$419.0657, found: 419.0648 .

Ethyl-2-(4-chlorophenyl)-1',3',4-trioxo-1',3'-dihydrospiro[cyclopentane-1,2'-indene]-3carboxylate (3d). White solid, mp 127.5-129.1 ${ }^{\circ} \mathrm{C} ;{ }^{1} \mathrm{H} \mathrm{NMR}\left(400 \mathrm{MHz}, \mathrm{CDCl}_{3}\right) \delta$ : 7.90 (d, J 7.2 $\mathrm{Hz}, 1 \mathrm{H}), 7.78-7.50(\mathrm{~m}, 3 \mathrm{H}), 7.06-7.00(\mathrm{~m}, 4 \mathrm{H}), 4.42$ (d, J 13.6 Hz, 1H), 4.37 (d, J 14.0 Hz, 1H), 4.19-4.14 (m, 2H), 2.97 (d, J 18.4 Hz, 1H), 2.68 (d, J $18.4 \mathrm{~Hz}, 1 \mathrm{H}), 1.23(\mathrm{t}, J 7.6 \mathrm{~Hz}, 3 \mathrm{H}) ;{ }^{13} \mathrm{C}$ NMR (100 MHz, $\left.\mathrm{CDCl}_{3}\right) \delta: 204.3,201.8,199.7,167.2,142.2,141.4,136.4,136.3,134.0,132.4$, $129.0,128.9,123.4,123.2,61.9,60.0,57.8,52.0,44.1,14.1$; IR (thin film) $v / \mathrm{cm}^{-1}: 2925(\mathrm{w})$, 1704(w), 1596(s), $1561(\mathrm{~s}), 1353(\mathrm{~m}),, 1088(\mathrm{~m}), 996(\mathrm{~m}), 862(\mathrm{~m})$; HRMS (ESI) calcd for $\mathrm{C}_{22} \mathrm{H}_{17} \mathrm{ClNaO}_{5}(\mathrm{M}+\mathrm{Na})^{+}: 419.0657$, found: 419.0653 .

Ethyl-2-(2-methoxyphenyl)-1',3',4-trioxo-1',3'-dihydrospiro[cyclopentane-1,2'-indene]-3carboxylate (3e). Light yellow solid, mp 124.1-125.8 ${ }^{\circ} \mathrm{C} ;{ }^{1} \mathrm{H}$ NMR $\left(400 \mathrm{MHz}, \mathrm{CDCl}_{3}\right) \delta$ : 7.91 (d, J 7.6 Hz, 1H), 7.72 (br, 1H), 7.61 (br, 1H), 7.54 (d, J 7.6 Hz, 1H), 7.10 (d, J 7.6 Hz, 1H), 6.99 (br, 1H), 6.79 (br, 1H), $6.36(\mathrm{~d}, J 8.0 \mathrm{~Hz}, 1 \mathrm{H}), 4.73$ (d, J 14.0 Hz, 1H), 4.45 (d, J 13.6 Hz, 1H), 4.17-4.14 (m, 2H), 3.49 (s, 3H), 3.10 (d, J 18.8 Hz, 1H), 2.73 (d, J $18.8 \mathrm{~Hz}, 1 \mathrm{H}), 1.22$ (t, J 7.2 $\mathrm{Hz}, 3 \mathrm{H}) ;{ }^{13} \mathrm{C}$ NMR $\left(100 \mathrm{MHz}, \mathrm{CDCl}_{3}\right) \delta: 205.9,201.7,198.3,167.4,156.7,142.8,140.7,135.5$, $135.4,129.0,127.0,122.7,122.3,121.9,120.5,109.5,61.7,59.5,57.4,54.1,46.4,42.4$, 14.1; IR (thin film) $v / \mathrm{cm}^{-1}: 1705(\mathrm{w}), 1598(\mathrm{~s}), 1562(\mathrm{~s}), 1432(\mathrm{~m}), 1354(\mathrm{~m}), 1075(\mathrm{~m})$; HRMS (ESI) calcd for $\mathrm{C}_{23} \mathrm{H}_{20} \mathrm{NaO}_{5}(\mathrm{M}+\mathrm{Na})^{+}: 415.1152$, found: 415,1146

Ethyl-2-(4-methoxyphenyl)-1',3',4-trioxo-1',3'-dihydrospiro[cyclopentane-1,2'-indene]-3carboxylate (3f). Light yellow solid, mp 112.8-114.2 ${ }^{\circ} \mathrm{C} ;{ }^{1} \mathrm{H}$ NMR $\left(400 \mathrm{MHz}, \mathrm{CDCl}_{3}\right) \delta: 7.88$ $(\mathrm{d}, J 1.6 \mathrm{~Hz}, 1 \mathrm{H}), 7.75-7.72(\mathrm{~m}, 3 \mathrm{H}), 6.98(\mathrm{~d}, J 8.8 \mathrm{~Hz}, 2 \mathrm{H}), 6.58(\mathrm{~d}, J 8.8 \mathrm{~Hz}, 2 \mathrm{H}), 4.41(\mathrm{~d}, J$ $13.6 \mathrm{~Hz}, 1 \mathrm{H}), 4.35$ (d, $J 13.6 \mathrm{~Hz}, 1 \mathrm{H}), 4.18-4.13(\mathrm{~m}, 2 \mathrm{H}), 3.62(\mathrm{~s}, 3 \mathrm{H}), 2.97$ (d, $J 18.4 \mathrm{~Hz}, 1 \mathrm{H})$, 2.67 (d, J $18.4 \mathrm{~Hz}, 1 \mathrm{H}), 1.22$ (t, J $6.8 \mathrm{~Hz}, 3 \mathrm{H}) ;{ }^{13} \mathrm{C} \mathrm{NMR}\left(100 \mathrm{MHz}, \mathrm{CDCl}_{3}\right) \delta: 205.1,202.2$, 200.1, 167.5, 159.2, 142.3, 141.6, 136.1, 136.0, 128.7, 125.6, 123.2, 123.1, 113.9, 61.7, 60.1, 58.0, 55.0, 52.4, 43.9, 14.1; IR (thin film) $v / \mathrm{cm}^{-1}:$ 1704(w), 1562(s), $1515(\mathrm{~s}), 1354(\mathrm{~m}), 1076$ (m), 862 (m); HRMS (ESI) calcd for $\mathrm{C}_{23} \mathrm{H}_{20} \mathrm{NaO}_{5}(\mathrm{M}+\mathrm{Na})^{+}: 415.1152$, found: 415.1145 . 
Ethyl-2-(4-fluorophenyl)-1',3',4-trioxo-1',3'-dihydrospiro[cyclopentane-1,2'-indene]-3carboxylate (3g). Light yellow solid, mp 154.7-156.5 ${ }^{\circ} \mathrm{C} ;{ }^{1} \mathrm{H} \mathrm{NMR}\left(400 \mathrm{MHz}, \mathrm{CDCl}_{3}\right) \delta: 7.91$ $(\mathrm{d}, J 6.8 \mathrm{~Hz}, 1 \mathrm{H}), 7.80-7.75(\mathrm{~m}, 3 \mathrm{H}), 7.06-7.00(\mathrm{~m}, 4 \mathrm{H}), 4.42(\mathrm{~d}, J 13.6 \mathrm{~Hz}, 1 \mathrm{H}), 4.37(\mathrm{~d}, J 14.0$ $\mathrm{Hz}, 1 \mathrm{H}), 4.19-4.14(\mathrm{~m}, 2 \mathrm{H}), 2.97$ (d, J 18.4 Hz, 1H), 2.68 (d, J $18.4 \mathrm{~Hz}, 1 \mathrm{H}), 1.23$ (t, J 7.2 Hz, $3 \mathrm{H}) ;{ }^{13} \mathrm{C}$ NMR (100 MHz, $\left.\mathrm{CDCl}_{3}\right) \delta: 204.3,201.8,199.7,167.2,142.2,141.4,136.4,136.3$, 134.0, 132.4, 129.0, 128.8, 123.4, 123.2, 114.0, 112.4, 61.9, 60.0, 57.8, 52.0, 44.1, 14.1; IR (thin film) $v / \mathrm{cm}^{-1}:$ 1704(w), 1599(s), $1562(\mathrm{~s}), 1433(\mathrm{~m}), 1354(\mathrm{~m}), 1075$ (m), 863 (m); HRMS (ESI) calcd for $\mathrm{C}_{22} \mathrm{H}_{17} \mathrm{FNaO}_{5}(\mathrm{M}+\mathrm{Na})^{+}$: 403.0952, found: 403.0963 .

Ethyl-2-(4-bromophenyl)-1',3',4-trioxo-1',3'-dihydrospiro[cyclopentane-1,2'-indene]-3carboxylate (3h). White solid, mp 144.9-146.5 ${ }^{\circ} \mathrm{C} ;{ }^{1} \mathrm{H}$ NMR (400 MHz, $\left.\mathrm{CDCl}_{3}\right) \delta: 7.83(\mathrm{~d}, J 6.4$ $\mathrm{Hz}, 1 \mathrm{H}), 7.71-7.68(\mathrm{~m}, 3 \mathrm{H}), 7.13(\mathrm{~d}, J 8.4 \mathrm{~Hz}, 2 \mathrm{H}), 6.88(\mathrm{~d}, J 8.4 \mathrm{~Hz}, 2 \mathrm{H}), 4.34$ (d, J 13.6 Hz, $1 \mathrm{H}), 4.29(\mathrm{~d}, J 14.0 \mathrm{~Hz}, 1 \mathrm{H}), 4.12-4.07(\mathrm{~m}, 2 \mathrm{H}), 2.89$ (d, J $18.4 \mathrm{~Hz}, 1 \mathrm{H}), 2.61$ (d, J $18.4 \mathrm{~Hz}, 1 \mathrm{H})$, $1.16(\mathrm{t}, J 6.8 \mathrm{~Hz}, 3 \mathrm{H}) ;{ }^{13} \mathrm{C} \mathrm{NMR}\left(100 \mathrm{MHz}, \mathrm{CDCl}_{3}\right) \delta: 204.3,201.7,199.7,167.2,142.2,141.4$, $136.4,136.3,132.9,131.8,129.3,123.4,123.3,122.2,61.9,59.6,57.7,52.0,44.2$, 14.1; IR (thin film) $v / \mathrm{cm}^{-1}:$ 1704(w), 1598(s), $1563(\mathrm{~s}), 1433(\mathrm{~m}), 1354(\mathrm{~m}), 1057$ (m), 863 (m); HRMS (ESI) calcd for $\mathrm{C}_{22} \mathrm{H}_{17} \mathrm{BrNaO}_{5}(\mathrm{M}+\mathrm{Na})^{+}:$463.0152, found: 463.0144, 465.0134.

Ethyl-1',3',4-trioxo-2-(thiophen-2-yl)-1',3'-dihydrospiro[cyclopentane-1,2'-indene]-3carboxylate (3i). Yellow solid, mp 112.4-113.8 ${ }^{\circ} \mathrm{C} ;{ }^{1} \mathrm{H} \mathrm{NMR}\left(400 \mathrm{MHz}, \mathrm{CDCl}_{3}\right) \delta: 7.96(\mathrm{~d}, J$ $7.2 \mathrm{~Hz}, 1 \mathrm{H}), 7.83-7.77(\mathrm{~m}, 3 \mathrm{H}), 6.94(\mathrm{~d}, J 4.8 \mathrm{~Hz}, 1 \mathrm{H}), 6.76(\mathrm{~d}, J 3.2 \mathrm{~Hz}, 1 \mathrm{H}), 6.69$ (t, J 4.8 Hz, $1 \mathrm{H}), 4.66(\mathrm{~d}, J 13.2 \mathrm{~Hz}, 1 \mathrm{H}), 4.35(\mathrm{~d}, J 13.2 \mathrm{~Hz}, 1 \mathrm{H}), 4.25-4.16(\mathrm{~m}, 2 \mathrm{H}), 2.97(\mathrm{~d}, J 18.4 \mathrm{~Hz}, 1 \mathrm{H})$, $2.68(\mathrm{~d}, J 18.4 \mathrm{~Hz}, 1 \mathrm{H}), 1.26(\mathrm{t}, J 6.8 \mathrm{~Hz}, 3 \mathrm{H}) ;{ }^{13} \mathrm{C} \mathrm{NMR}\left(100 \mathrm{MHz}, \mathrm{CDCl}_{3}\right) \delta: 204.0,201.7$, $199.5,167.1,142.5,141.6,136.6,136.2,136.1,126.8,126.3,125.0,123.4,123.3,61.9$, 59.7, 59.6, 48.0, 44.1, 14.1; IR (thin film) $v / \mathrm{cm}^{-1}: 1705(\mathrm{w}), 1597(\mathrm{~s}), 1561(\mathrm{~s}), 1433(\mathrm{~m}), 1384(\mathrm{~m})$, $1076(\mathrm{~m}), 619(\mathrm{~m})$; HRMS (ESI) calcd for $\mathrm{C}_{22} \mathrm{H}_{16} \mathrm{SNaO}_{5}(\mathrm{M}+\mathrm{Na})^{+}$: 391.0611, found: 391.0605 .

Ethyl-1',3',4-trioxo-2-(thiophen-2-yl)-1',3'-dihydrospirocyclopentane-1,2'-indene (4). White

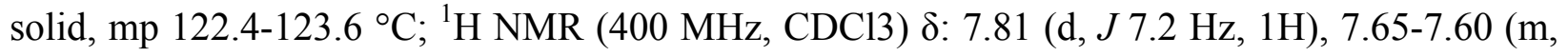
$3 \mathrm{H}), 6.99-6.95(\mathrm{~m}, 5 \mathrm{H}), 3.94$ (q, $J 13.6 \mathrm{~Hz}, J 8.0 \mathrm{~Hz}, 1 \mathrm{H}), 3.27-3.24$ (br, 1H), 2.82 (d, J $18.4 \mathrm{~Hz}$, 1H), 278-2.66 (br, 1H), 2.56 (d, J $18.4 \mathrm{~Hz}, 1 \mathrm{H}) ;{ }^{13} \mathrm{C}$ NMR (100 MHz, CDCl3) $\delta: ~ 201.1,200.0$, $197.0,141.2,140.6,135.0,134.8,134.2,127.5,126.9,126.5,122.1,122.0,61.1,49.3,43.2$, 40.7; IR (thin film) v/cm-1: 1704(w), 1587(s), 1563 (s), 1431(m), 1380 (m), 619(m); HRMS (ESI) calcd for $\mathrm{C}_{19} \mathrm{H}_{14} \mathrm{NaO}_{3}(\mathrm{M}+\mathrm{Na})^{+}: 313.0835$, found: 313.0845 .

\section{Acknowledgements}

This work was financially supported by Research Program of Science and Technology from Education Department of Jiangxi Province, China (No.GJJ14717) and National Natural Science Foundation of China (Nos. 21172270, 21202208). 


\section{References}

1. Kuan, B.; Hao, H.; Han, C. C.; Kwunmin, C. Org. Lett. 2013, 15, 2880. http://pubs.acs.org/doi/abs/10.1021/o14011689

2. Maheswari, S. U.; Balamurugan, K.; Perumal, S.; Yogeeswari, P.; Sriram, D. Bioorg. Med. Chem. Lett. 2010, 20, 7278.

http://www.sciencedirect.com/science/article/pii/S0960894X10015416

3. Stefan, T. A.; Florian, H.; Seeliger, B. Org. Biomol. Chem. 2007, 5, 3020. http://pubs.rsc.org/en/content/articlelanding/2007/ob/b708025e\#!divAbstract

4. Dai, B. F.; Song, L. P.; Wang, P. Y.; Yi, H.; Cao, W. G.; Jin, G. F.; Zhu, S. Z.; Shao, M. Synlett 2009, 1842.

https://www.thieme-connect.com/products/ejournals/abstract/10.1055/s-0029-1217361

5. Shchepin, V. V.; Stepanyan, Y. G.; Silaichev, P. S. Russian Journal of General Chemistry 2008, 78, 5, 929 .

http://link.springer.com/article/10.1134/S1070363208050162

6. Evans, P. A.; Thomas, A. B. Tetrahedron Lett. 1996, 37, 1367. http://www.sciencedirect.com/science/article/pii/004040399600055X

7. Pizzirani, D.; Roberti, M.; Recanatini, M. Tetrahedron Lett. 2007, 48, 7120. http://www.sciencedirect.com/science/article/pii/S0040403907015596

8. Chai, Z.; Rainey, T. J. J. Am. Chem. Soc. 2012, 134, 3615. http://pubs.acs.org/doi/abs/10.1021/ja2102407

9. Yavari, I.; Mokhtarporyani-Sanandaj, A.; Moradi, L. Tetrahedron Lett. 2007, 48, 6709. http://www.sciencedirect.com/science/article/pii/S0040403907014360

10. Kitson, P. J.; Parenty, A. D. C.; Richmond, C. J.; Long, D.-L.; Cronin, L. Chem. Commun. 2009, 4067. http://pubs.rsc.org/en/content/articlelanding/2009/cc/b905383b\#!divAbstract

11. Pizzirani, D.; Roberti, M.; Grimaudo, S.; Cristina, A. D.; Pipitone, R. M.; Tolomeo, M.; Recanatini, M. J. Med. Chem. 2009, 52, 6936. http://pubs.acs.org/doi/abs/10.1021/jm900907s

12. Ramachary, D. B.; Barbas III, C. F. Chem. Eur. J. 2004, 10, 5323. http://onlinelibrary.wiley.com/doi/10.1002/chem.200400597/abstract

13. Li, M.; Yang, W.-L.; Wen, L.-R.; Li, F.-Q. Eur. J. Org. Chem. 2008, 2751. http://onlinelibrary.wiley.com/doi/10.1002/ejoc.200800035/abstract

14. Ramachary, D. B.; Venkaiah, C.; Krishna, P. M. Chem. Commun. 2012, 48, 2252. http://pubs.rsc.org/en/content/articlelanding/2012/cc/c2cc17219d

15. Ramachary, D. B.; Anebouselvy, K.; Chowdari, N. S.; Barbas III, C. F. J. Org. Chem. 2004, 69, 5838.

http://pubs.acs.org/doi/abs/10.1021/jo049581r

16. Gyulmalieva, T. M.; Ismailova, V. M.; Yusubova, N. N.; Moskvab, V. V. Russ. J. Org. Chem. 2009, 45, 12, 1862. 
http://link.springer.com/article/10.1134/S1070428009120203

17. Ren, Z.-J.; Cao, W.-G.; Tong, W.-Q.; Chen, J.; Deng, H.-M.; Wu, D.-Y. Synth. Commun. 2008, 38, 2200.

http://www.tandfonline.com/doi/full/10.1080/00397910802029406\#.VDSfPq6jhug

18. Russo, A.; Meninno, S.; Tedesco, C.; Lattanzi, A. Eur. J. Org. Chem. 2011, 5096. http://onlinelibrary.wiley.com/doi/10.1002/ejoc.201100562/abstract

19. Luo, N.-H.; Sun, X.; Yan, Y.-Y.; Nie S.-Z.; Yan M. Tetrahedron:Asymmetry 2011, 22, 1536. http://www.sciencedirect.com/science/article/pii/S0957416611004897

20. Yin, X.-G.; Liu, X.-Y.; Hu Z.-P.; Yan M. Org. Biomol. Chem. 2012, 10, 1506. http://pubs.rsc.org/en/content/articlelanding/2012/ob/c2ob06995d\#!divAbstract

21. Li, X.-M.; Wang, B.; Zhang J.-M.; Yan M. Org. Lett. 2011, 13, 374. http://pubs.acs.org/doi/abs/10.1021/o1102570b

22. Hu Z.-P.; Lou, C.-L.; Wang, J.-J.; Chen C.-X.; Yan. M. J. Org. Chem. 2011, 76, 3797. http://pubs.acs.org/doi/abs/10.1021/jo200112r

23. Wang, J.-J.; Hu Z.-P.; Lou, C.-L.; Liu, J.-L.; Li X.-M.; Yan M. Tetrahedron 2011, 67, 4578. http://www.sciencedirect.com/science/article/pii/S0040402011006235

24. Dong, L.-T.; Du, Q.-S.; Lou, C.-L.; Zhang J.-M.; Lu R.-J.; Yan M. Synlett. 2010, 266. https://www.thieme-connect.com/products/ejournals/abstract/10.1055/s-0029-1218570

25. Zhang J.-M.; Hu Z.-P.; Zhao S.-Q.; Yan M. Tetrahedron 2009, 65, 802. http://www.sciencedirect.com/science/article/pii/S004040200802005X

26. Zhang J.-M.; Hu Z.-P.; Dong, L.-T.; Xuan Y.-N.; Yan M. Tetrahedron: Asymmetry 2009, 20, 355.

http://www.sciencedirect.com/science/article/pii/S0957416609000342

27. Xuan Y.-N.; Nie, S.-Z.; Dong, L.-T.; Zhang J.-M.; Yan M. Org. Lett. 2009, 11, 1583. http://pubs.acs.org/doi/abs/10.1021/o1900227j

28. Lee, C. J.; Sheu, C. N.; Tsai, C. C.; Wu, Z. Z.; Lin, W. W. Chem. Commun. 2014, 50, 5304. http://pubs.rsc.org/en/content/articlelanding/2014/cc/c3cc45201h 